Tome 40, no 28. - Juillet 1933.

Prof. Dr. E. HANDSCHIN

Studienreise auf den Sundainseln und in Nordaustralien. 1930-1932.

\title{
Odonaten aus Nordaustralien
}

von

\section{A. LIEFTINCK}

(Zoologisches Museum, Buitenzorg.)

Mit 8 Textfiguren.

Die von Herrn Prof. Ed. Handschin während seiner Forschungsreise in Nord-Australien vom April bis zum Juni 1931 und im April 1932 gesammelten Odonaten wurden mir nach Heimkehr des Reisenden in liebenswürdigster Weise zur Bearbeitung anvertraut.

Sämtliche Arten stammen aus dem wasserarmen Küstengebiet südwestlich und südlich von Port Darwin, Nord-Australien, also aus einer Gegend, deren Insektenfauna noch recht lückenhaft bekannt war.

Die vorliegende Sammlung setzt sich aus nicht weniger als 39 Arten zusammen, wovon zwar die meisten gewöhnlichen oder doch weit verbreiteten Formen angehören; ein Teil ist aber von wesentlichem Interesse, da er einerseits einige Unklarheiten in der Literatur beseitigen half und andrerseits unsere Kenntnis der australischen Libellenfauna ergänzt und vermehrt. Wichtig ist in erster Linie der Fund der Hemicordulia intermedia, dann aber auch derjenige der Austrogynacantha heterogena, einer vielleicht nicht dem eigent lichen Urwaid angehörigen Art.

Die Typen der neuen Arten Notoneura fraterna und Isosticta handschini, nebst dem Weibchen der Hemicordulia intermedia befinden sich zusammen mit einer Belegserie der übrigen Arten in den zoologischen Sammlungen des Museums von Basel; ein Teil

Rev. Suisse de Zool., T. 40, 1933. 
der Sammlung befindet sich in den Sammlungen des Museums in Canberra und in denen des Verfassers.

\section{ZYGOPTERA.}

Fam. Lestidae.

1. Lestes paludosus Tillyard.

M a t e ri a l : 2 ㅇ (semiad.), Z.-Lagoon, 19.IV.1931; 1 우 (def.), Kadarri, IV.1931; 1 우 (ad.), Marraki, 16.V.1931.

Leider fehlt das $\hat{o}$, so dass die Bestimmung etwas fraglich bleiben muss. Die Exemplare stimmen gut mit den Beschreibungen überein, sind aber der javanischen concinnus Selys ausserordentlich ähnlich.

Das $ᄋ$ von Marrakai ist sehr schön in den Farben erhalten. Die ganze Färbung ist licht gelbbraun, und die Thoraxseiten sind gleichmässig licht bläulichweiss bereift.

\section{Lestes albicaudus McLachlan.}

M a t e ri a l: 1 ㅇ (juv.), Marrakai, 15.V.1931.

Kleiner und zierlicher als die vorige Art und sehr leicht $\mathrm{zu}$ unterscheiden an der in beiden Flügeln verschieden gestalteten $q$. Die metallgrüne Längslinie jederseits parallel zur Mediannaht des Thorax ist noch nicht voll entwickelt, aber doch ganz deutlich erkennbar.

\section{Fam. Agrionidae.}

Subfam. Protoneurinae.

3. Notoneura fraterna sp. n.

(Fig. 1.)

M a t e ri a l: 4 ô, 5 우 (ad.), Z.-Lagoon, 19.IV, und 4 ô, 2 우 (juv.), Adelaide River, 21.IV.1931.

Gehört in die von Ris (1913) zum ersten Male abgetrennte papuanisch-australische Gruppe II, die dadurch gekennzeichnet ist, 
dass das Rudiment von $C u_{1}$ im Hinterflügel nicht die Verlängerung der ersten posttetragonalen Querader erreicht.

Sehr ähnlich N. solitaria (Till.), von N. Queensland.

ô (ad.). - Kopf gross, stark behaart. Unterlippe gelb, am Ende geschwärzt. Kopf tiefschwarz bis auf eine schmale, in der Mitte oft unterbrochene und seitlich etwas verbreiterte gelblich-grüne Querbinde der Stirn, vorne den Postclypeus berührend, hinten von der Fühlerwurzel aber weit entfernt bleibend. Seitlich von den lateralen Ozellen je ein winziger rötlicher Punkt.

Prothorax ganz schwarz; Lobus posterior ein wenig aufgerichtet, in flachem Kreisbogen begrenzt.

Synthorax kurz und ziemlich robust, oben und seitlich lang schwarz behaart. Dorsum, bis zur vorderen Seitennaht, tief schwarz, mit äusserst schmalen, hell gelblichgrünen, unvollständigen Antehumeralstreifen, den Flügelsinus oben nicht erreichend und ventralwärts schon etwa im Niveau der Hinterstigmen aufhörend. Seiten hell gelblich-grün, mit einer breiten, tiefschwarzen Binde über die hintere Seitennaht; diese Binde ist genau so breit wie die metepisternale gelbe Binde (deren oberes Ende etwas vom dorsalen Rande abgerückt und dort gerundet ist). Metepimerum, unteres Drittel des Metinfraepisternum und Ventralfläche gelblichgrün.

Coxae gelb, aussen diffus bräunlich gefleckt. Beine ganz schwarz, bis auf einen ganz schmalen gelben Basalring der Femora. Dornen schwarz; Klauenzähne deutlich.

Flügel kurz, Membran entweder gänzlich hyalin (juv.), oder mit ziemlich starker Gelbfärbung, von der Basis bis etwas über den Nodus hinaus und sehr diffus nach aussen abschliessend. Nervatur wie solitaria. Pnq $\frac{10-11}{8-9}$. Pterostigma schief, nahezu parallelseitig, ungefähr zweimal länger als breit, braunschwarz.

Abdomen sehr schlank und dünn. Schwarz, bronzeglänzend. Segm. 1 seitlich breit, 2 etwas schmaler licht grünlich; 2 überdies mit scharf ausgebildeter, hell grünlichgelber Linie über die vordere Hälfte der Mediannaht. Segm. 3-7 an der Basis mit ganz schmalen, aber vollständigen, hell grünlichgelben Basalringeln, die sich unten eine kurze Strecke entlang den Tergiträndern fortsetzen. Segm. 8-10 völlig schwarz, bis auf einen sehr deutlich ausgeprägten, hell grünlichgelben Streifen, jederseits entlang des Seitenrandes des Tergites: dieses Segment also nur am Unterrande gefärbt. 
Appendices sehr kurz, in der Form ähnlich Nososticta solida (Tillyard, 1913, pl. 46, fig. 3-4), wie in der Fig. 1 dargestellt. Basale Hälfte der superiores schwarz, sonst hellgelb. Appendices inferiores schwarz.

q (ad.). - Kopf wie $\hat{\jmath}$, die Stirnbinde aber viel breiter gerade, und überall gleich breit, nach hinten bis halbwegs zur Fühlerbasis reichend, hellgelb. Punktflecken jenseits der Ozellen grösser, rötlichgelb.

Prothorax schwarz, nahe dem Unterrande schmal gelb gesäumt und oberseits mit grossen, paarigen, gelben Flecken auf den Buckeln des mittleren Abschnittes. Lobus anterior lappig aufgerichtet, in der Mitte kurz und fein eingekerbt. Lobus posterior

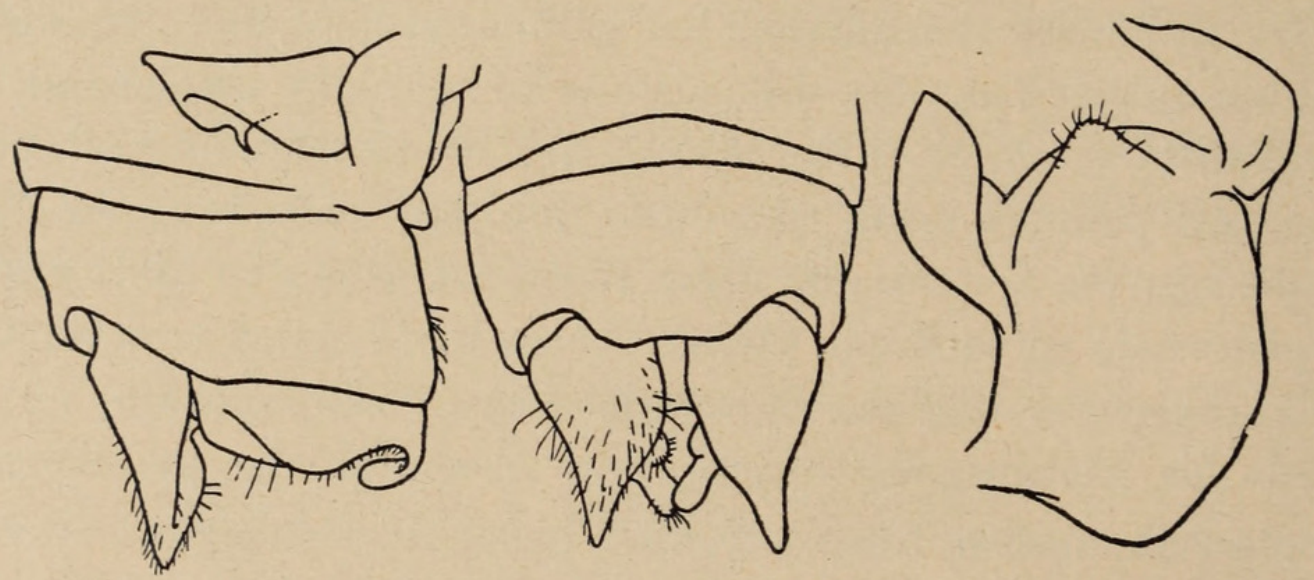

FIG. 1. - Notoneura fraterna, sp. n.

Appendices des $\hat{\sigma}$, von rechts und dorsal, rechte App. sup. schräg von unten rechts (oben) und Prothorax des $q$ von links.

sehr gross und aufgerichtet, von oben gesehen ist das nach vorne umgeklappte Endteil concav und bis zur Spitze stark dreieckig verschmälert (Fig. 1).

Thorax wie beim $\hat{o}$ gefärbt, aber die antehumerale Linie ist breiter, parallelseitig und nahezu complett, ohne jedoch den Antealarsinus nach aufwärts zu erreichen. Schwarze Binde über die hintere Seitennaht etwas mehr reduziert und schmäler als die gelbe metepisternale Binde.

Beine schwarz. Basale Hälfte der Femora und Streckseiten des 2. und 3. Tibienpaares bräunlichgelb.

Flügel hyalin. $P n q \frac{9-11}{9-10}$. 
Abdomen sehr schlank, Mittelsegmente jedoch kräftiger und cylindrisch; Segm. 8-10 nur wenig erweitert. Gefärbt wie beim §ే; die basalen Punktfleckchen vom 3.-6. Segment in der Mitte fein unterbrochen, sehr hell gelb. Tergitränder bis knapp vor dem Ende ziemlich breit grünlichgelb. Segm. 8-10 oberseits ganz schwarz, aber mit breiter grünlicher Linie am Seitenrand. Sternite schwarz.

Appendices schwarz, etwas abgeflacht, spitz, ungefähr ebenso lang als das sehr kurze 10. Segment. Valven den Appendix kaum überragend, der Unterrand sehr leicht gebogen und am Ende äusserst fein gezähnelt.

Länge: §ิ Abdomen + Appendices 25-26, Hinterflügel $15 \mathrm{~mm}$.; ㅇ 25,5-26, 16-16,5 $\mathrm{mm}$.

Von der Gattung Notoneura, deren Hauptverbreitungsgebiet in Neu Guinea liegt, waren bis jetzt nur zwei Arten, solitaria Till. und coelestina Till., aus Australien bekannt. Beide Arten sind angeblich bisher nur im Norden Queenslands aufgefunden worden.

Nach den Angaben von Tillyard (1906) und Ris (1913) lassen die Männchen sich nun leicht folgendermassen unterscheiden:

\section{Bestimmungs-Tabelle der ổ.}

1. Appendices superiores in der Seitenansicht gerade abgestutzt, die Fläche eben ein wenig ausgehöhlt, ganz hellblau. Breite, seitlich erweiterte, grünblaue Stirnlinie. Antehumeralstreifen länglich oval, vorn am breitesten, olivengrün. An den Seiten des 8. Abdominalsegments ein länglicher grünlicher Fleck; 9-10 schwarz. Abd. 30, Hfl. 19 mm. . . . . . . . . . . . . . coelestina.

1'. Appendices superiores in der Seitenansicht von gewöhnlicher Form, d.h. dreieckig bis zum Ende verschmälert und einfach spitz, schwarz oder teilweise gelblich. Schmale, gerade, oder ein wenig seitwärts erweiterte, grüngelbe Stirnlinie. Antehumeralstreifen sehr schmal, nach unten nicht erheblich verbreitert, gelb oder grünlichgelb. . . . 2

2. Vordere Hälfte des 8. Abdominalsegments mit grosser hellgrüner Zeichnung. Appendices superiores konisch, abgeflacht, und einfach spitz, ohne zahnartige Bildungen. Abdomen 26-28, Hinterflügel $17.5 \mathrm{~mm}$ solitaria

2'. 8. Abdominalsegment nur unten am Seitenrande mit schmalem, hellgelben Längsfleck. Appendices superiores wie solitaria, die Spitze jedoch schlanker und mehr ausgezogen; scharfer, nach unten und hinten gerichteter median-ventraler Zahn. Abd. 25-26, Hfl. 15 mm

fraterna. 


\section{Austrosticta fieldi Tillyard.}

1908. Tillyard, Proc. Linn. Soc. N. S. Wales (1907), V. 32, p. $765-$

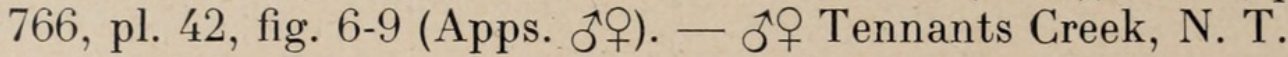

1913. Tillyard, Ibid. (1912), V. 37, p. 434-435, pl. 46, fig. 13-14 (Apps. §̋), 47 fig. 5 (Flügel). - Gleiche Ex.

1917. Sıösted t, Arkiv f. Zool., 11: 29-31, pl. 3, fig. 5-9 (Apps. §̊우). ઠ우 Kimberley Distr., N. W. T. (A. soror).

M a t e ri a l: $3 \widehat{\diamond}, 1$ ㅇ, Kadarri, Burnside und Z. Lagoon, 19.V.1931; 2 ô, Burnside, 22.IX-3.V.1932.

Diese sehr eigentümliche, strohgelb und metallisch grün gefärbte Art liegt in einigen gut ausgewachsenen Stücken vor. Die Tiere
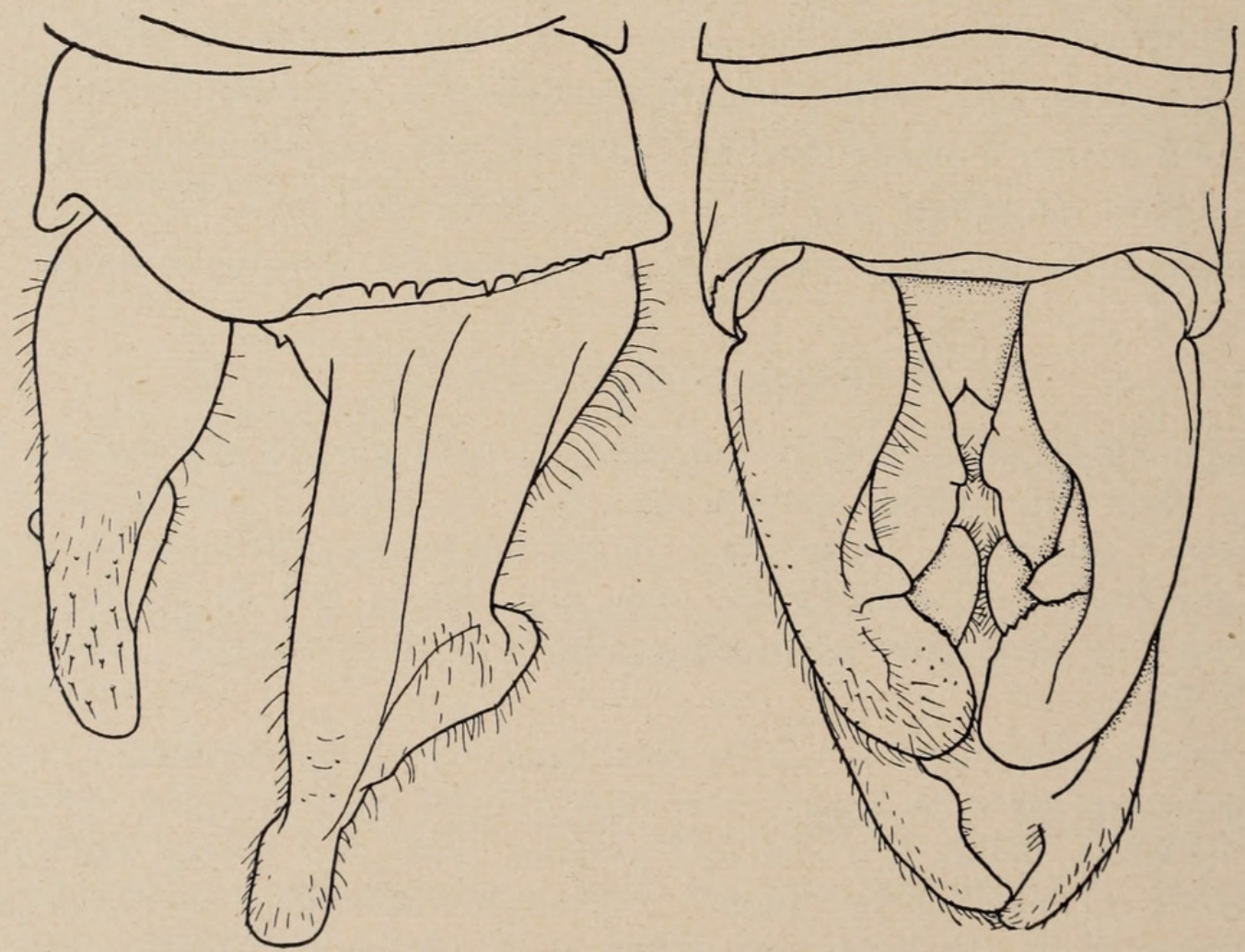

FIG. 2. - Austrosticta fieldi Tillyard, ô.

Appendices von rechts und von oben.

stimmen in allen Einzelheiten mit der Originalbeschreibung überein, nur wäre zur Ergänzung der Genusdiagnose hervorzuheben, dass die Tarsalklauen einen deutlichen subapikalen Zahn aufweisen, und dass die Querader $C u q$ manchmal der 1. Anq ganz nahe gerückt erscheint. Die schmale, metallische Seitenbinde des Thorax ist in 
ihrem dorsalen Ende öfters längsgeteilt und die gelbe Humerallinie am unteren Ende bisweilen schmal gebrochen. Am ô ProthoraxHinterrande ist $\mathrm{zu}$ ersehen, dass der paarige, beiderseits etwas wulstig erhabene Mittellappen jederseits in ein stumpf abgerundetes Häckchen endet. Beim + ist der Hinterrand völlig gerundet und unbewehrt. Ursprung der Sektoren $M_{2}$ und $M_{1 \mathrm{a}}$ in beiden Flügeln variabel. Postnodalqueradern $\frac{9}{7-8}$. Pterostigma länger und schmaler als in der Figur bei Tillyard (l.c.).

Am ô Abdomen fällt das schwärzlich gefärbte 8. und 9. Sternit wie auch ein schwarzer Randstreifen am 9. Tergit auf.

Die eigenartig gestalteten männlichen Appendices habe ich noch einmal sorgfältig abgebildet, um zu zeigen, dass dieselben in der Form eine Mittelstellung einnehmen zwischen den von Tillyard und Sлӧsтерт abgebildeten Zangenpaaren. Es ist nicht daran zu zweifeln, dass Sנöstedt's soror mit unserer Art identisch ist. Die genannten (und illustrierten) Unterschiede sind geringfügiger Natur und werden ausnahmslos durch die mir jetzt vorliegende Serie überbrückt.

A. fieldi, wenn auch offenbar eine sehr seltene Art, scheint also in Nordaustralien eine weite Verbreitung zu haben.

Grösse ein wenig variabel: ô Abdomen + Appendices 26,5-29, Hinterflügel 18-20; ㅇ 28, $21 \mathrm{~mm}$.

\section{Genus Isosticta Selys.}

Als ich (1932) die aberrante Gattung Stenosticta, mit ihren beiden Arten filiformis (Ris), vom Bismarck-Archipel, und fissicollis Lieft. vom nördlichen Neu Guinea, untersuchte, wurde ein eingehenderes Studium aller vorläufig in Isosticta untergebrachten Spezies des östlichen Inselgebietes und von Australien beabsichtigt.

Wenn ich auch heute noch von der Notwendigkeit überzeugt bin, einmal die drei neu-caledonischen Isosticta-Arten, spinipes Selys, robustior Ris, und tillyardi Campion von den übrigen Arten des australischen Festlandes generisch zu trennen, so haben sich bei der exacten Diagnostisierung dieser geographisch gut getrennten Gruppen so erhebliche Schwierigkeiten ergeben, dass man auf Grund der Flügelnervatur und unter der Voraussetzung, die Trennungsmerkmale konsequent durchzuführen, unbedingt ge- 
nötigt wurde, zuerst die neu-caledonische robustior von den ebenfalls dort lebenden tillyardi und spinipes generisch $\mathrm{zu}$ trennen. Dieses Verfahren würde uns aber folgerichtig auch zwingen, die beiden übrig gebliebenen Arten spinipes und tillyardi der australischen simplex-Gruppe gegenüber zu stellen. Wenn es für die Aufstellung eines neuen Genusnamen genügte, nur ein einziges freilich recht charakteristisches - Merkmal zu benützen (in diesem

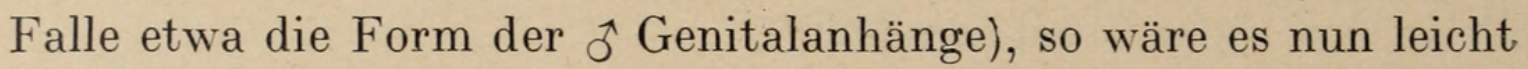
möglich, die schmächtigere und düster gefärbte simplex-Gruppe abzugrenzen. Wenn Campion ${ }^{1}$ aber im Anfang seiner allgemeinen Bemerkungen zu Isosticta sagt: "It is not possible from the literature alone to make a complete comparison between them in respect of the labium, the hind margin of the prothorax, and the tibial armature, but, as will be gathered from the following table, they do not present any great uniformity in certain venational characters of importance ", dann muss ich ihm durchaus zustimmen. Es wäre nämlich ganz besonders hervorzuheben, dass I. spinipes und tillyardi hinsichtlich der Flügeladerung ziemlich genau eine Mittelstellung zwischen robustior einerseits und der simplex-Gruppe andererseits einzunehmen scheinen. Die erheblichen Unterschiede in der Lage von $R s$ und $M_{3}$ bei $I$. spinipes und deren übrigens recht nahen Verwandten robustior und tillyardi veranlassen mich, derartige, sonst vielfach recht nützliche Merkmale mit grösster Vorsicht zur Trennung einzelner Gattungen zu verwenden. Eben die unvollständige Auskunft der Beinbedornung und Prothorax-Armatur bei den australischen Vertretern hindert mich, an dieser Stelle näher auf die Genusberechtigung der verschiedenen Arten einzugehen. Jedenfalls genügt Campion's knapp gefasste Gruppenverteilung vollständig zur raschen Orientierung in den Verwandschaftsverhältnissen.

Charakteristisch für alle bis jetzt bekannten Arten ist die ausserordentlich schlanke Gestalt, die düstere, wenigstens teilweise metallische Färbung des Körpers, die modifizierte Form des männlichen Prothorax, die deutlich gezähnten Tarsalklauen, die weit proximal von der ersten $A n q$ gelegenen $C u q$, und die Konfiguration der Appendices anales.

1 Cf. Campion, Ann. Mag. Nat. Hist., 8-9th Ser. (1921), p. 37. 
Die Unterschiede Stenosticta gegenüber sind beträchtlich und lassen sich folgendermassen zusammenfassen:

1. Flügel deutlich gestielt, die Erweiterung im Niveau des Arculus oder etwas proximal oder distal desselben liegend; distale Fläche normal und verhältnissmässig breit. Ursprung von $M_{3}$ höchstens um ein wenig distal vom Subnodus und $R s$ höchstens in der Mitte zwischen Subnodus und $P n q_{1}$ entspringend. $C u_{1}$ wenigstens eine Zelle lang; $M_{4}$ sehr deutlich analwärts gebrochen. Beine ziemlich kräftig, Femora 3 den Hinterrand des Metepimerum kaum erreichend; Dornen kurz und kräftig. Pterostigma mit der costalen Seite länger als die anale, die distale Seite mässig bis sehr schief, mit spitzer Aussenecke. Körper des $\hat{o}$ ad. wenigstens teilweise mit blauer Bereifung, schwarz oder mit geringem Bronzeglanz. Thorax kurz und gedrungen, Abdomen sehr schlank. Appendices superiores des $\widehat{o}$ von gleicher Länge oder kürzer als inferiores. Australien, Neu-Caledonien . . . . . . . . . . . . . . . Isosticta Sel.

1'. Flügel aus sehr schmaler Basis ganz allmählich distalwärts erweitert, die Fläche ausserordentlich schmal. Ursprung von $M_{3}$ ungefähr in der Mitte zwischen Subnodus und $P n q_{1}$ gelegen, $R s$ an oder etwas distal von denselben entspringend. $C u_{1}$ eine Zelle lang; $M_{4}$ nahe dem Flügelrande um einige Zellen undeutlich gebrochen. Pterostigma rhombisch, die costale Seite etwas kürzer als die anale, und die distale Seite gerade oder eben ein wenig zurückgebogen, die Aussenecke also nicht spitz. Beine dünn und sehr lang, die Femora 3 das Ende des 1. Abdominalsegments erreichend. Dornen sehr kurz und fein. Körper ohne Bereifung, lebhaft erzgrün und hellgelb. Thorax und Andomen filiform. Appendices superiores des $\hat{o}$ viel länger als die inferiores. Neu Guinea, Bismarck Archipel . . . . . . . . . Stenosticta Lft.

\section{Isosticta handschini, sp. n.}

(Fig. 3-4.)

M a t e ri a l: 1 ô ad., Shoebridge, 18.IV.1931; 1 q juv., Kadarri, 20.IV.1931; 2 ๙ิ ad., Burnside, 21.IV.1932.

Nahe I. banksi Till., aber leicht verschieden durch geringere Grösse, das Vorhandensein je eines Hornfortsatzes am Hinterrande des $\hat{o}$ Prothorax und durch die erheblich verschieden gestalteten Appendices. Im übrigen gleich.

ô (ad.). - Kopf oben und vorn mattschwarz. Clypeus etwas glänzend; Oberlippe glänzend schwarz, sehr breit hellgelb gerandet. Genæ und Mandibelbasis ebenfalls gelb. Zweites Fühlerglied gelbbraun, am Ende angedunkelt. Oberseite des Kopfes schwach bläulich bereift. 
Prothorax sehr dunkel erzgrün, ziemlich dicht kreideartig bereift; Form aus der Figur 3 ersichtlich; Hinterlappen deutlich abgegrenzt, aber nicht aufgerichtet, jederseits am Hinterrande mit einem flach seitwärts gerichteten und sehr leicht vorwärts gekrümmten, starken Horn, das am Ende ziemlich stark lateral zusammengedrückt erscheint.

Synthorax oben und seitlich ebenso gefärbt wie der Prothorax, schwach grünmetallisch, ohne lichte Zeichnung, nur am ventralen
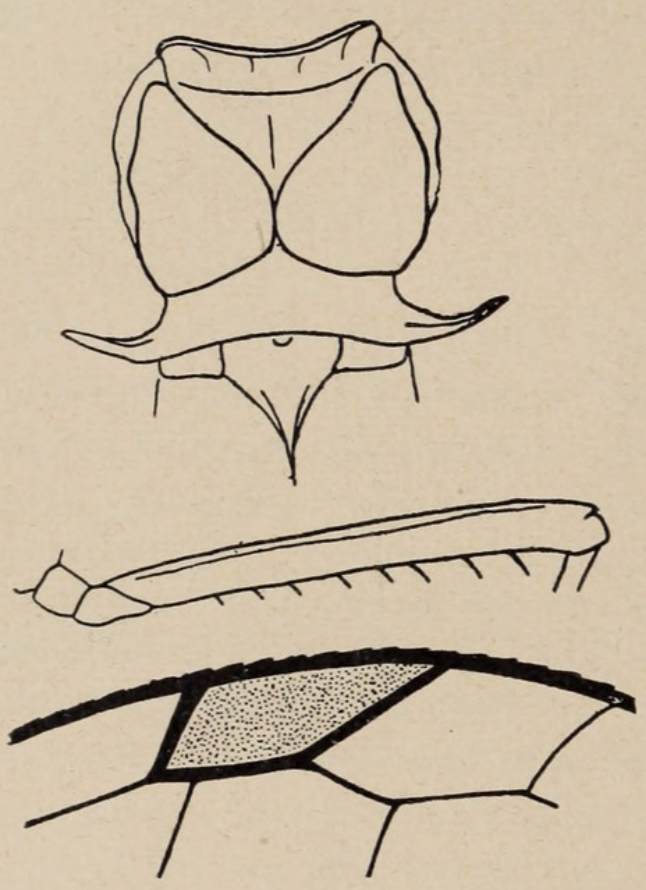

FIG. 3.

Isosticta handschini, sp. n., $\widehat{\jmath}$.

Prothorax von oben gesehen, Hinterfemur und Pterostigmaregion eines Hinterflügels.

Ende der hinteren Seitennaht eine gelbbraune metepisternale Aufhellung, wie auch unten am Rande des Metepimerum. Ventralseite blass gelb. Bereifung dünn, aber sehr gleichmässig, nur am unteren Seitenrand etwas dichter.

Coxen gelblich, mit je einem dunklen Mittelfleck. Bereifung ziemlich grob. Beine ziemlich kurz und kräftig; Femora bräunlichgelb, aussen mit einer nach unten breiteren dunklen Strieme, die am ersten Beinpaar auf die untere Hälfte beschränkt ist. Tibia gelb, innen mit einer schwarzen Linie. Tarsen gelb, die Glieder am Ende schwarz; Klauen mit schwarzer Spitze und sehr kurzem, annäherend rechteckigen Zahn. Dornen kurz und kräftig (Fig. 3), Femora 1-2, Femora 2-3, 7-9.

Flügel ähnlich $I$. banksi; $P n q \frac{13-14}{11}$. Ursprung von $M_{3}$ am oder etwas proximal vom Subnodus. $C u_{1}$ im Vorderflügel etwas über eine halbe Zelle lang, im Hinterflügel bis zu der ersten posttetragonalen Querader reichend und am Flügelrande aufhörend (also wie bei simplex und banksi). Pterostigma ziemlich lang und sehr schief, schwarzbraun, sehr fein gelblich gesäumt.

Abdomen gefärbt wie banksi, aber viel kürzer; Segm. 1-2 oben bereift; von Segm. 7 an ganz verdunkelt und ebenfalls mit blauer Bereifung.

Appendices superiores cylindrisch, schlanker und stärker ein- 
wärts gekrümmt als bei banksi, bis zum Ende allmählich verschmälert und vollständig abgerundet; ein subapikaler Höcker ist kaum angedeutet. Appendices inferiores mit einem sehr deutlichen, nach hinten bis zwei Drittel ihrer Länge erreichenden, stumpf rechteckigen, inneren Fortsatz, der am Ende unregelmässig gezähnelt ist und in der Seitenansicht als eine gerundete Wölbung vorspringt; schwarz.
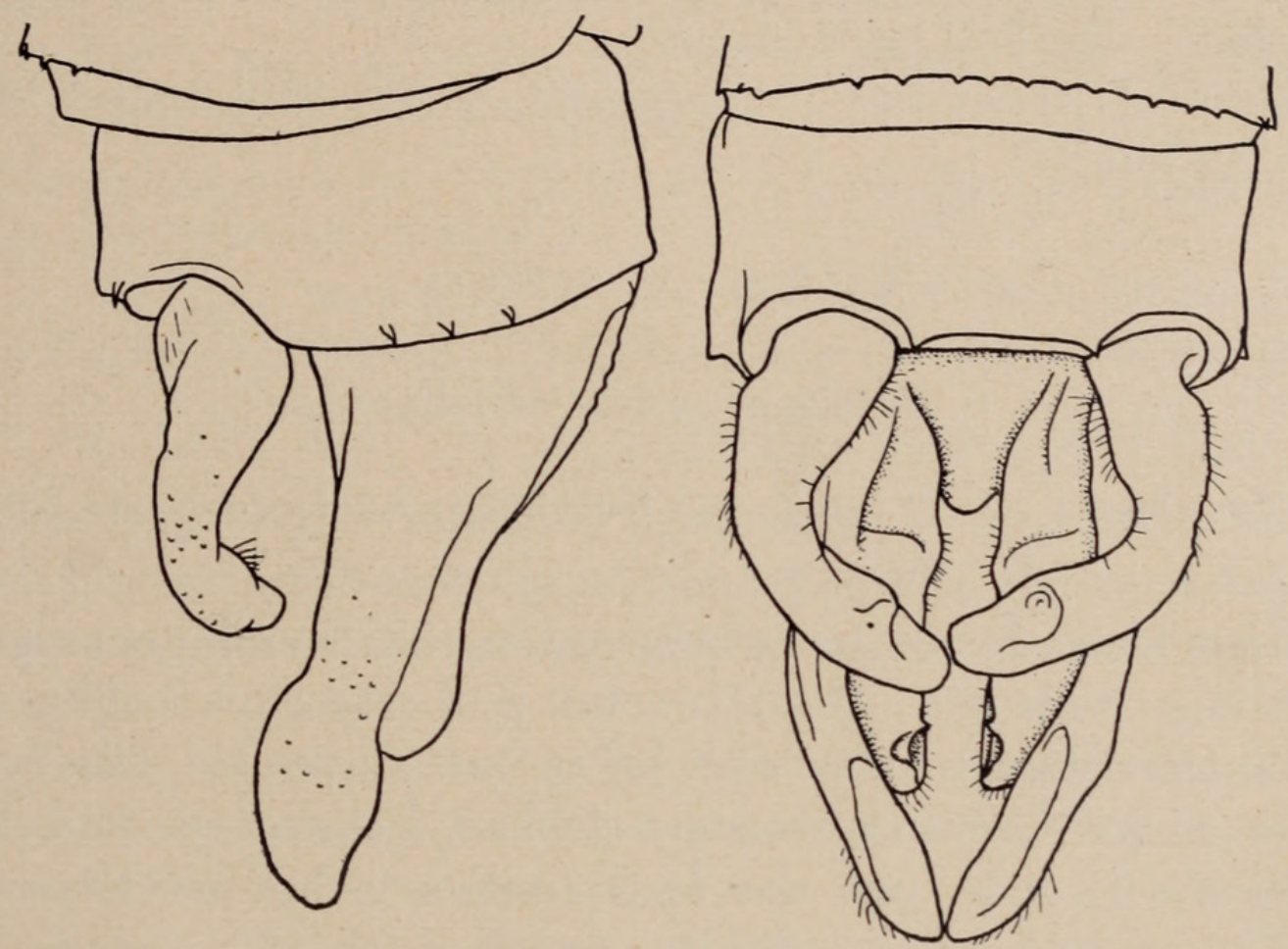

FIG. 4. - Isosticta handschini, sp. n., ô. Appendices von rechts und von oben.

† (juv.). - Körper weisslich- oder bräunlichgelb; die dunklen Zeichnungen ziemlich stark grünglänzend. Dem ô sehr ähnlich, aber verschieden durch das Vorherrschen der lichten Partien.

Oberlippe kaum dunkler an der Basis, Postclypeus mit zwei gelben Flecken jederseits der Mitte. Vorderlappen des Prothorax ganz, und zwei Längsstreifen auf der Mitte, blassgelb; Hinterlappen erzgrün, seitlich vollständig abgerundet. Thorax oberseits metallisch grün, mit fein gelblicher Mittellinie und einem etwas breitern, geraden Humeralstreifen, der sich ganz nahe dem Oberrand mit der hellen Farbe der Seiten verbindet. Metepisternum mit schmalem, unten bis zum Stigma reichenden dunklen Streifen. Seiten übrigens blass gelblich oder fleischfarben, wie auch die ganze Sternalfläche. Beine blass gelblich. 
Flügel mit $\frac{15-15}{12-12} P n q$. Im Vorderflügel erreicht $C u_{1}$ den Flügelrand an der ersten posttetragonalen Querader. $M_{3}$ deutlich proximal vom Subnodus. Pterostigma blassgelb.

Abdomen cylindrisch (in der Form nicht gut erhalten); terminale Segmente dick und robust; die gelbe Färbung längs der Seiten des Hinterleibes ist deutlicher, so dass nur ein Band längs der Mitte des Rücken metallisch grün bleibt. Valven das Ende des 10. Segmentes deutlich überrangend.

Länge: ๙ิ Abdomen + Appendices, 30-31; Hinterflügel 18-19; 우 $28,19 \mathrm{~mm}$.

\section{Subfam. Agrioninae.}

\section{Pseudagrion ignifer Tillyard.}

M a t e ri a l: 3 ô (ad.), 4 ㅇ (semiad.), Adelaide River, 21.IV. 1931.

Unsere kleine Serie stimmt in allen Einzelheiten mit der Originalbeschreibung überein. Die Männchen sind sehr gut erhalten. Die Antehumeralstreifen und das lichte metepisternale Band sind jedoch nicht "brown", sondern lebhaft grünlichgelb, auf tiefschwarzem Grunde, und das Metepimerum nebst der Unterseite des Thorax sind schön lichtblau bereift.

Diese Art fliegt nur an fliessendem Wasser (Tillyard, Handschin) und steht auch in dieser Hinsicht zweifellos P. crocops Selys (Philippinen, Celebes, Molukken) am nächsten.

\section{Pseudagrion microcephalum (Ramb.).}

\section{(P. australasiae Till., nec Selys.)}

M a t e ri a l : $1 \hat{\jmath}$ (ad.), Adelaide River, 21.IV und $1 \hat{\jmath}, 1$ \& (ad.) Katherine, 5.VI.1931.

Ueber den blauen Pseudagrion des östlichen Faunengebietes habe ich vor kurzem in einer Arbeit über papuanische Libellen ausführlicher berichtet und dort auch die jetzt vorliegenden Stücke kurz erwähnt und besprochen (1932, p. 576).

Die australische microcephalum wurde bereits von Ris (1916) und SJöstedT (1917) unter dem richtigen Namen aufgeführt und be- 
schrieben. Eine sehr gute Abbildung der Appendices anales des $\hat{\sigma}$ hat zum ersten Male Tillyard (1913) gegeben.

\section{Ceriagrion erubescens Selys.}

(Fig. 5.)

1891. Selys, Ann. Mus. civ. Genova, V. 10 (30), p. 84-85. - Queensland etc. ( $C$. coromandelianum race erubescens).

1906. Tillyard, Proc. Linn. Soc. N. S. Wales, V. 31, p. 191. Brisbane, Queensland (C. glabrum).

1913. Tillyard, Ibid., V. 37, p. 471, pl. 49, fig. 17-18 (Apps.). -

N. Queensland (C. glabrum).

1913. Ris, Abh. Senckenb. Naturf. Ges. 34.2: 519 (Tabelle), 520, T. 23, fig. 13 (Flügel), 14 (Apps.). - Aroe, N. Guinea, Formosa.

M a te ri a l: 2 ô, 1 ㅇ (ad.), Darwin, VI und 9.VI.1931.

Die wichtigste Literatur über diese unter verschiedenen Namen aufgeführte Art ist oben aufgezählt und die vielen kleineren Notizen in Faunenwerken habe ich absichtlich nicht erwähnt, weil sie nichts Neues bringen.

Jetzt bin ich in der Lage, die mir früher immer noch etwas zweifelhaft erscheinenden Stücke von Nord-Australien mit dem weit verbreiteten $C$. erubescens in regelrechte Beziehung $\mathrm{zu}$ bringen, wie es schon vor Jahren durch Ris geschah und neuerdings auch von TILLYARD (1926) und mir (1932) angenommen wurde. Durch die Güte des Herren A. BALL konnte ich eins der von $\mathrm{DE}$ SELYs erwähnten $\widehat{\jmath}$ aus Queensland und ein ô aus Shanghai (beide im Museum Brüssel auf-

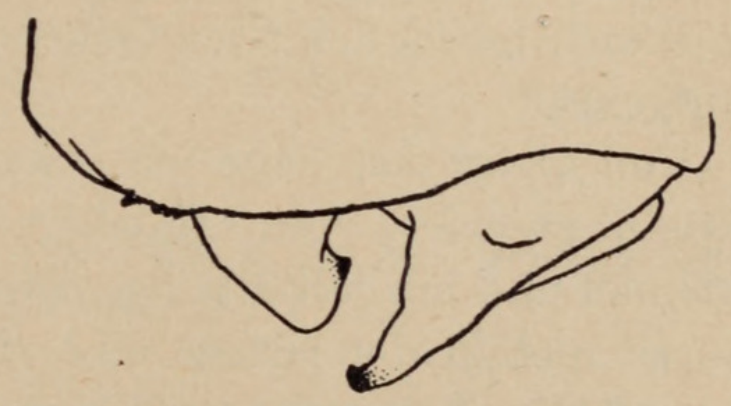

Fig. 5 .

Ceriagrion erubescens Selys.

๙ิ Type, Queensland (Mus. Brüssel).

Appendices in Seitenansicht. bewahrt) untersuchen und eine ganze Serie von roten Ceriagrion aus Sumatra, Java, den Aroe Inseln und Neu-Guinea vergleichen.

Die Art ist von coromandelianum durchaus verschieden und eigentlich unverkennbar, hat aber mit bellona Laidlaw (Borneo) und inaequidens Lieft. (Neu-Guinea) vieles gemeinsam, vor allem die Färbung. Sehr charakteristisch ist die Konfiguration am Hinter- 
rande des 10. Abd.-Segmentes und die Form der Appendices superiores.

Unsere Exẻmplare sind völlig identisch mit dem SELys'schen $\widehat{0}$ aus Queensland. Das $\hat{\jmath}$ aus Shanghai, in der Färbung ganz gleich, zeigt als einzigen Unterschied etwas verkürzte Appendices inferiores, die genau so lang sind als das obere Paar. Ich gebe noch eine Skizze nach dem Queensland Exemplar zum Vergleich mit den von Ris und Tillyard (loc. cit.) gegebenen Abbildungen.

\section{Agriocnemis rubescens Selys.}

1877. Selys, Synopsis Agrion (suite et fin), p. $42-43$ (Sep.). + Queensland.

1900. Ris, Arch. f. Naturg., 1, Taf. 10, Fig. 19 (Apps., Queensland).

1913. Tillyard, Proc. Linn. Soc. N. S. Wales, 37, p. 454-455, pl. 49, fig. 3-4 (Apps.), fig. 5 (ô Proth.). - Queensland, N. S. Wales.

1930. Ris, Arkiv. f. Zool, 21A: 10-12, Fig. 4 (Apps. Luzon). Philippinen, Aroe, Cape York.

1932. Lieftinck, Nova Guinea, 15, Zool. 5, p. 588 (Spezies-Tabelle). Sumatra, Java, etc.

Eine vollständige Synonymie bis 1913 findet sich bei Ris (1913). Die wichtigsten Schriften früheren und späteren Datums sind oben aufgezählt.

Von dieser Art habe ich etwa 300 Stück aus dem weiten Verbreitungsgebiet, von Malakka bis Australien, genau ansehen können und bin mit Ris der Meinung, dass die A. rubescens strukturell und in der Grösse eine ziemlich variable, aber immer gut kenntliche Art repräsentiert. An Exemplaren von Malakka, Sumatra, Java, Borneo, den Philippinen, Celebes und von der Insel Flores war ich nicht imstande, mehr als eine Art zu unterscheiden. A. rubescens variiert sehr stark in der Grösse. Die kleinsten Exemplare, die ich gesehen, stammen aus Borneo und Nord Australien, die grössten von West Java und Celebes ${ }^{1}$. Gut ausgefärbte

${ }^{1}$ Westjavanische rubescens sind im Gebirge viel grösser als in niederen Lagen, und die Männchen erreichen dort ein mehr vollendetes Stadium der Ausfärbung (vollständig schwarz mit dichter kreidigweisser Bereifung der Thoraxseiten und Abdomen!) als sonst in den Küstengegenden. Strukturdifferenzen treten dabei kaum zutage, obwohl geringe Unterschiede in der Form des Prothorax-Hinterrandes in beiden Sexen gefunden wurden, die aber nicht ausreichen, um eine selbständige Gebirgsart abzutrennen. 
Männchen der verschiedenen Inseln sehen einander überraschend ähnlich, und greifbare Unterschiede habe ich nicht finden können.

Neuerdings ist mir eine zweite und deutlich verschiedene Spezies aus dem nördlichen Neu Guinea bekannt geworden, deren Artberechtigung mir zweifellos erscheint, sowohl im Vergleich mit westlichen Argiocnemis als mit der Form aus Nordaustralien. Sie lässt sich sofort an der Prothorax-Armatur und den Appendices erkennen.

M a t e r i a l : 4 ㅇ, Kadarri, 20.IV.1931, 1 §ै, 1 q (ad.), Burnside, 21.IV.1932.

Die Stücke von Kadarri und Burnside sind sehr klein, aber sonst identisch mit den übrigen untersuchten Exemplaren. Eines der jungen Weibchen von Kadarri hat einen licht bräunlich-roten Thorax, völlig ohne dunkle Zeichnung; eine derartige Form des 우 habe ich in der Literatur noch nicht erwähnt gefunden. Tillyard's Figuren (l. c. 1913) sind recht instruktiv; nur wäre darauf hinzuweisen, dass seine Figur 3 auf Tafel 49 der männlichen Appendices offenbar in schräger Stellung (von hinten oben) gezeichnet worden ist.

10. Austroagrion exclamationis Campion.

(Fig. 6.)

1915. Campion, Ann. Mag. Nat. Hist. (8), t. 16, p. 105-108, fig. (Apps.). - ố Koolpinyah und Darwin, N. T.

M a t e ri a l : 2 ô (ad.), Burnside, IV und 2.V.1931; 1 우 (ad.), Bullrun-Swamp, 20.IV.1931; 2 오 (semiad.), Marrakai, 21.V.1931.

Diese sehr zierliche Agrionide liegt in einigen tadellosen Stücken vor. Die Type (British Museum) stammen aus der gleichen Gegend und unterscheiden sich in nichts Wesentlichem von den frischen Stücken der neuen Ausbeute.

Zur Ergänzung der ausführlichen Originalbeschreibung sei noch bemerkt, dass die schmalen Postocularflecken beim $\hat{\sigma}$, wie die Stirn, grün gefärbt sind. $P n q \frac{7-8}{6-7}$. Die bronzegrünen Längsstreifen auf dem Rücken des 3.-5. Abdominalsegments sind vor dem Ende stark eingeschnürt, aber nie unterbrochen. 
Appendices superiores schwarz, mit trübgelber Spitze; inferiores an der Basis licht braun, am Ende schwarz. Der innere schwarze Zahn der Appendices superiores ist für diese Art recht charakteristisch (Fig. 6).
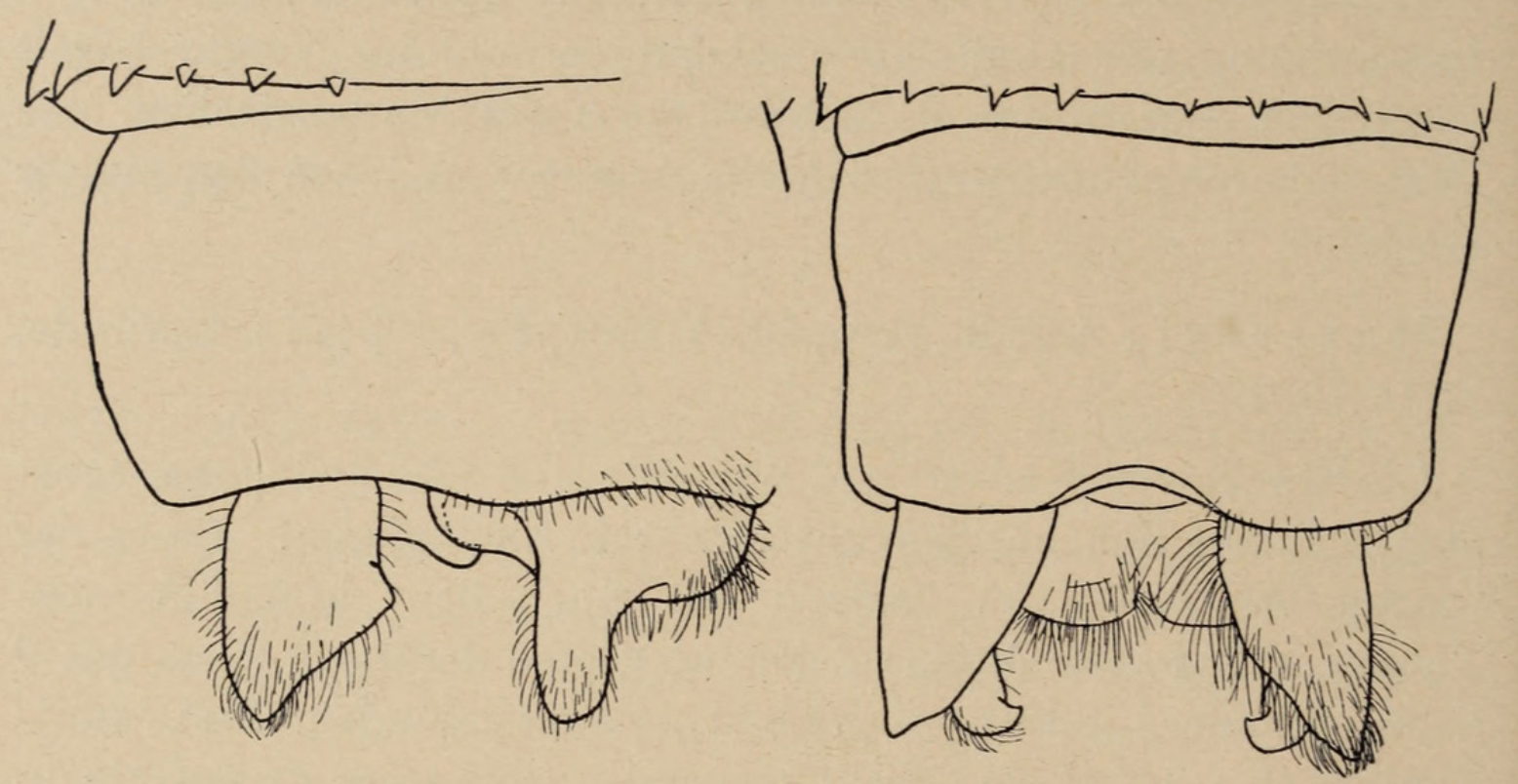

Fic. 6. - Austroagrion exclamationis Campion, $\widehat{\jmath}$.

Appendices von rechts und von oben.

Beim $ᄋ$ ist der Hinterrand des Prothorax tief dreilappig, der Mittellappen schmal dreieckig.

\section{Aciagrion fragilis (Tillyard).}

(Fig. 7.)

M a t e ri a l: 4 ô (ad.), Z.-Lagoon, 19.IV.1931; 3 ô, 2 우, Kadarri, 20.IV.1931; 2 ฮै, Burnside, 2.V.1931 und 29.IV. 1932.

Leider bin ich nicht in der Lage, diese Exemplare mit typischen fragilis zu vergleichen, obgleich mir deren Bestimmung ziemlich sicher vorkommt. Mehr als eine Art ist übrigens aus Australien noch nicht bekannt geworden.

Eine neue Figur der $\hat{\jmath}$ Appendices herzustellen, hat sich als Notwendigkeit ergeben, da die Tillyard'sche Zeichnung (1913) eine etwas andere Vorstellung der Anhänge gibt als die unsrige. 
Ich habe die kleine Serie mit einer viel grösseren aus dem nördlichen Neu-Guinea verglichen und keine Unstimmigkeiten gefunden,
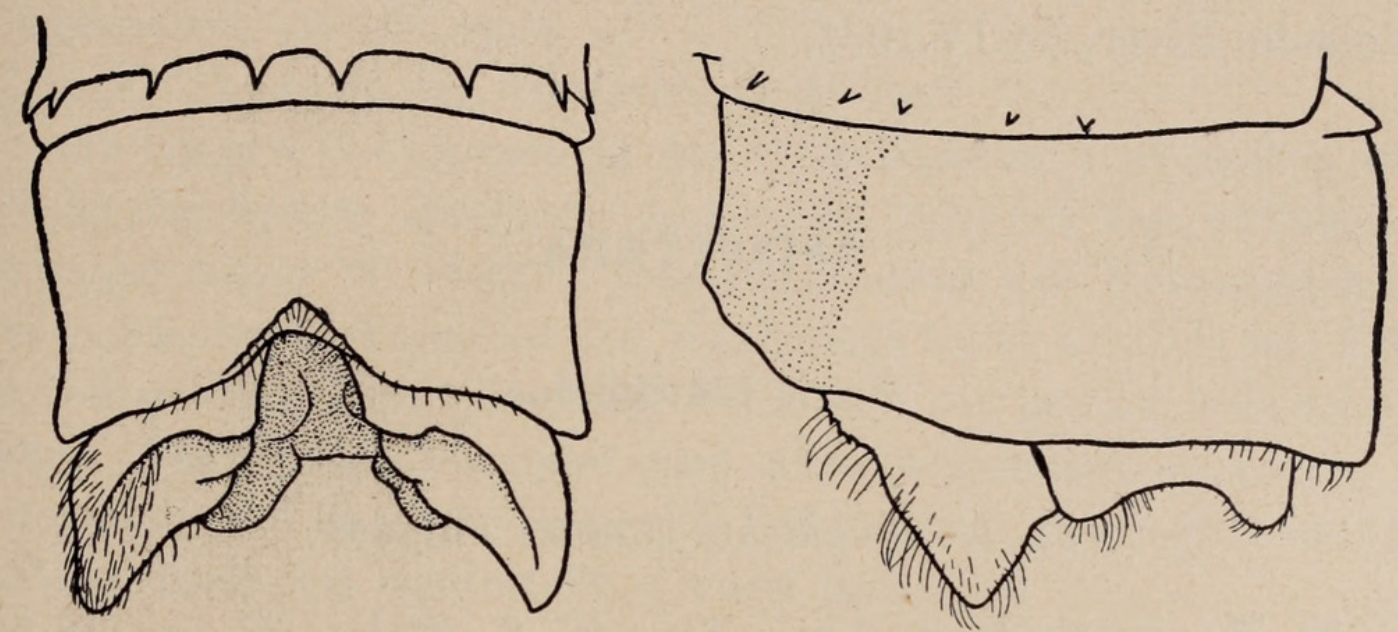

Fig. 7. - Aciagrion fragilis (Tillyard), $\hat{0}$

Appendices von oben und von rechts.

so dass ich annehme, dass $A$. fragilis auch in Papua vorkommt und somit eine weite Verbreitung zu haben scheint. ${ }^{1}$

\section{Ischnura torresiana Tillyard.}

1913. Tillyard, Proc. Linn. Soc. N. S. Wales, V. 37, p. 452-453, pl. 48, fig. 5-6 (Apps.). ợ Queensland.

1913. Ris, Abh. Senckenb. Naturf. Ges., Bd. 34, p. 5; 5 (Tabelle), 516,

Taf. 23, fig. 10 (Apps.). - Aroe, Neu Guinea, N. Queensland.

M a t e ri a l : 1 ๙ै, Kadarri, 20.IV.1931; 1 ô, 1 ㅇ, Burnside, 29.IV.1931 1 우, Adelaide River, 21.IV.1931; 2 §ै, Marrakai, 19.V. $1931 ; 4$ đૈ, 5 ㅇ, Darwin, Botanischer Garten.

\section{Ischnura aurora Brauer.}

M a t e r i a l : 2 ô (1 ad.), 1 \&, Z.-Lagoon, Kadarri und Bullrun Swamp, 19.-20.IV.1931; 2 ฮै, Marrakai und Burnside, 15-28.V. 1931; 2 우 Burnside, 22.IV.1932.

${ }^{1}$ Die Stücke von den Philippinen und Celebes wären noch genauer nachzuprüfen.

Rev. Suisse de Zool., T. 40, 1933. 
14. Agriocnemis pygmæa (Ramb.).

M a t e ri a l : 1 ô (semiad.), 15.V.1931; 1 ๆ (grüne Form), Adelaide River, 21.IV.1931.

\section{ANISOPTERA.}

\section{Fam. Libellulidae.}

\section{Nannophlebia eludens Tillyard.}

1908. Tillyard, Proc. Linn. Soc. N. S. Wales, 33, p. 645-647; pl. 14, fig. 4. - N. Queensland.

1913. Tillyard, Ibid. 37 (1912), p. 713, 715, etc. - N. Queensland

M a t e ri a l: 2 ㅇ (eins sehr juv.), Adelaide River, 21.IV.1931.

Das einzige in den Farben sehr gut erhaltene Weibchen lässt sich folgendermassen ergänzend beschreiben.

우 (ad.). - Kopf, Thorax und die basalen Hinterleibssegmente lichtgrün, mit braunschwarzen Zeichnungen. Segmente 3-7 mit orangefarbenen Ringen. Schwarze Stirnbasislinie äusserst schmal, nach unten längs des Augenrandes kaum fortgesetzt. Ein schwarzer Punkt in der Mitte der Frons. Thorax mit feiner Medianlinie die sich unten an der Quernaht zu einem rundlichen Flecken erweitert. Lichte Antehumeralbinde etwa anderthalb mal so breit als der dunkle Schulterstreif, erstere oben für eine kurze Strecke nach einwärts gekrümmt und entlang der Ante-alardreiecke sehr schmal, knapp vor der Mediannaht aufhörend. Dunkle Linie oberhalb an der hinteren Seitennaht äusserst schmal, etwas zackig und kaum mit dem breitern ventralen Teil zusammenhängend. Metasternum mit sehr schmalem dunklen Querstrich.

Flügel hyalin, ohne gelbe Basisflecken, die distale Fläche etwas bräunlichgelb angeflogen. $A n q \frac{6.6}{5.5}, P n q \frac{5.5}{5.5}$.

Abdomen mit vollständigem, grünlichgelbem Ring über die Mitte des 3. Segments, ziemlich genau das mittlere Drittel einnehmend. Segment 4 mit paarigem, oben nicht unterbrochenem und seitlich breit zusammenhängendem Ring über die Mitte, etwas hinter der 
Mitte des Segmentes; auf dem Rücken des 5. etwa ein Viertel, auf 6-7 ungefähr ein Drittel der Länge einnehmend. Segment 8 mit winzigem gelbem Seitenfleck und 9-10 schwarz.

Appendices orange, spitz.

Länge: Abdomen 20, Hinterflügel $21 \mathrm{~mm}$.

Trotz einigen Differenzen in der Flügelfärbung und in der Ausbildung der lichten Ringe am Abdomen, habe ich das vorliegende Weibchen unter $N$. eludens eingereiht unter der Voraussetzung, dass es bei der Unkenntnis des Männchens nicht möglich ist, diese Art sicher zu deuten. Einzelne Weibchen der papuanisch-australischen Nannophlebia-Gruppe sind ausserordentlich schwierig zu bestimmen und es wäre daher die Entdeckung des $\hat{\jmath}$ abzuwarten, ehe eine neue Art aufgestellt werden kann.

Vom australischen Festlande sind bisher nur zwei Arten bekannt, eludens und risi Tillyard (1913). Letztere Art ist viel grösser (Abdomen 23, Hinterflügel 23,5 mm.) und sieht überhaupt ganz anders aus wie eludens und deren papuanischen Verwandte.

Die vorliegenden zwei Weibchen nähern sich axiagasta Lieft. und ampycteria Lieft. von Nord-Neu Guinea an.

\section{Potamarcha obscura (Ramb.).}

1919. Ris, Cat. Coll. Selys, Lib., fasc. $16^{2}$ (1916), p. 1071. - ơㅇ Cape York.

M a te ri a l : 1 ㅇ (ad.), Burnside, 29.IV.1932.

Eine der häufigsten Libellulinen des indischen Archipels, nach dem Osten zu aber weit weniger allgemein und in Neu-Guinea sogar sehr selten.

Von Australien war nur das einzige von Ris beschriebene Pärchen bekannt. Das Weibchen der jetzigen Ausbeute ist in gleicher Weise sehr auffallend durch das Fehlen der dunklen Thoraxbinden, die hier so stark reduziert sind, dass nur noch ganz schmale dunkle Nähte übrig geblieben sind. Ganzer Thorax und Abdomen dünn blau bereift. Flügel völlig hyalin, ohne Spitzenfleck.

Dieser Rasse wäre weiter nachzugehen, ganz besonders im Trockengebiet der kleinen Sunda Inseln, wo sich Uebergänge zum insulären Typus des Westens finden dürften. Sonst wäre eine isoliert stehende Subspecies leicht $\mathrm{zu}$ definieren und abzutrennen. 


\section{Orthetrum caledonicum (Brauer).}

M a te ri a l : 3 ô, 1 + (ad.), Bullrun Swamp und Adelaide River, 20.-21.IV.1931; 3 ô, 1 우 (ad.), Katherine und Marrakai, 16.-17.V.1931.

Heimat: ganz Australien und Neu-Caledonien. Eine durch die hellblaue Bereifung des Körpers und die goldgefleckten Flügel sehr ausgezeichnete und schöne Art. O. caledonicum scheint in Neu-Guinea nicht vorzukommen, wurde jedoch neuerdings von Prof. Handschin auf der Insel Timor entdeckt, eine Tatsache die die engen faunistischen Beziehungen dieser Insel mit Australien aufs Neue bestätigt.

18. Orthetrum sabina (Drury).

M a te ri a l: $1 \hat{\jmath}$, Adelaide River, Kadarri, 21.IV.1931; 3 ऽิ, 1 ㅇ, Burnside und Marrakai, 2., 16.-20.V.1931.

19. Brachydiplax denticauda (Brauer).

M a t e r i a l : 2 ๙ (ad.), Marrakai, 17.V und Darwin, 9.VI.1931; 2 §ิ, 3 우 (semiad.), Burnside, 22.IV und 3.V.1932.

20. Nannodiplax rubra Brauer.

M a t e ri a l: 7 $\widehat{\jmath}, 7$ 오, Kadarri, Burnside, Z.-Lagoon, Bullrun Swamp, Adelaide River, 19.-29.IV.1931; 1 ๙ิ, Marrakai, 15.V.1931;

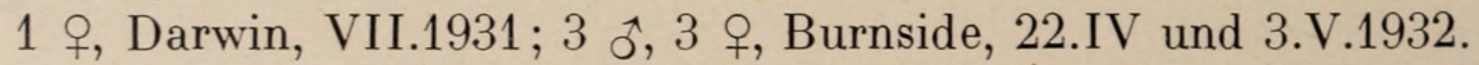

\section{Diplacodes nebulosa (Fabr.).}

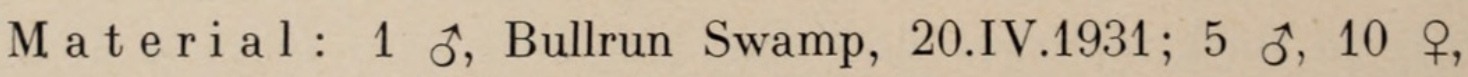
Burnside und Marrakai, 2., 15.-21.IV.1932.

22. Diplacodes trivialis (Ramb.).

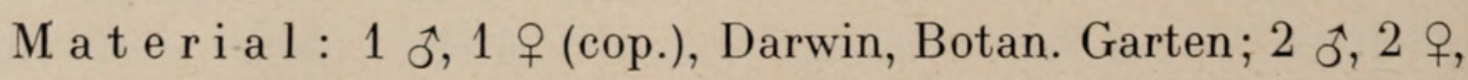
Burnside und Marrakai, 10.-15.V.1931. 


\section{Diplacodes bipunctata (Brauer).}

M a t e r i a l : 5 $\uparrow, 8$ ㅇ, Darwin, Burnside, Z.-Lagoon und Bullrun Swamp, 16.-29.IV.1931; 3 ô, 7 ㅇ, Marrakai und Katherine, 6., 15.-17.V.1931; 4 ô, 1 앙, Burnside, 21.-22.IV.1932.

\section{Diplacodes haematodes (Burm.).}

M a t e ri a l: 3 ô, 1 ㅇ, Burnside und Adelaide River, IV.1931;

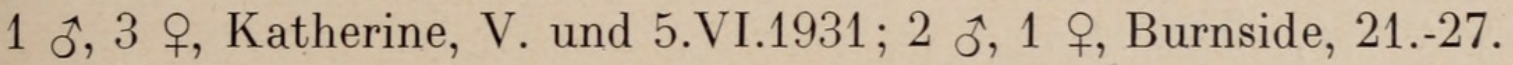
IV.1932.

Ein ausgefärbtes Männchen von Burnside wurde von Prof. Handschin einer Raubfliege (Asilide) entnommen.

\section{Crocothemis nigrifrons (Kirby).}

M a t e ri a l: $7 \hat{\jmath}$ (ad.), 1 q (juv.), Burnside, 15.-17.IV. und 2.V.1931; 1 ิ, 1 \&, Marrakai, 16.-17.V.1931; 1 §ิ, Adelaide River, 21.IV.1931; 1 ㅇ (ad.), Darwin, 9.VI.1931; 1 శ.

Grosse und robuste Exemplare. Das $q$ von Darwin ist voll ausgefärbt und ausgezeichnet durch ziemlich dichte blaue Bereifung des Abdomen.

Das von Ris (1911, p. 544) erwähnte q von Neu-Guinea gehört zweifellos zu dieser Art und nicht zu servilia, deren Vorkommen in Neu Guinea noch der Bestätigung bedürfte.

\section{Neurothemis stigmatizans stigmatizans (F.).}

M a t e r i a l: 10 ๙ै, 7 q (het.), Darwin (Bot. Garten), Shoebridge Camp, Z.-Lagoon, Burnside, Kadarri, IV.-V.1931; 1 o (het.), Burnside, 22.IV.1932.

Einige Individuen sind noch unausgefärbt; aber alle Weibchen gehören der heterochromen Form an, wie sie von Ris (1911) photographisch dargestellt wurde.

\section{Rhodothemis rufa (Ramb.).}

M a t e ri a l: 1 ô (ad.), 1 ㅇ (semiad.), Kadarri und Adelaide River, 20.-21. IV.1931; 1 ㅇ (ad.), Burnside, 3.V.1932. 
Relativ sehr kleine Exemplare mit auch beim adulten ${ }^{\tau}$ deutlich sichtbarer grünlichgelber Zeichnung auf dem Thoraxdorsum. Goldgelber (nicht goldbrauner) Basisfleck in Vorder- und Hinterflügel, bis $C u q$ im Hinterflügel reichend. Pterostigma hellgelb.

Weibchen dem $\hat{\sigma}$ in den Dimensionen ähnlich. Querbinde der Stirn sehr scharf abgegrenzt, hell grünlichgelb. Occiput, Prothorax, und zwei schmale, nahezu parallele Längsstreifen über die Thoraxmitte ebenso stark ausgeprägt, sehr hell gelbgrün.

Länge: $\widehat{\jmath}$, Abdomen + Appendices 23-24, Hinterflügel 29-30 mm; ㅇ, 22-24, 30-32 $\mathrm{mm}$.

Die in mancher Hinsicht abweichenden Exemplare der Ostverbreitung verdienen eine genauere strukturelle Untersuchung. Die oben erwähnten Exemplare aus Nord-Australien sind die kleinsten, die ich je gesehen habe und schliessen sich eng an die papuanische Form an. Auch die wenigen Stücke der kleinen Sunda-Inseln die ich untersuchen konnte, sind viel kleiner und schärfer gezeichnet als diejenigen von Sumatra und Java.

Die feurig roten Männchen aus West-Java sind im Fluge kaum von Crocothemis servilia Drury, und meistens überhaupt nicht von Urothemis signata bisignata Brauer zu unterscheiden.

\section{Zyxomma elgneri Ris.}

M a t e ria l: 1 ô (ad.), Darwin (Bot. Garten), 13.IV.1931; 2 ô (ad.), Burnside, 16.IV.1931.

Ursprünglich von Cape York und den Aroe Inseln beschrieben, und nachher auch von Tillyard in Brisbane (Queensland) gefunden.

Gleich gross und überhaupt völlig übereinstimmend mit der Originalbeschreibung. Eins der Männchen zeigt auf dem Thoraxdorsum und an den Seiten des 2. Abdominalsegmentes eine äusserst dünne, zart bläulich-violette Bereifung.

Ich erhielt eine grössere Serie dieser eigentümlichen Art vom nördlichen Neu-Guinea, so dass sie offenbar eine weitere Verbreitung hat als bisher angenommen wurde. Fliegt abends von Sonnenuntergang bis tief in die Nacht hinein sehr rasch über offenem Wasser, in Neu-Guinea zusammen mit $Z$. petiolatum Ramb., wo sie aber weit seltener ist. 


\section{Tholymis tillarga (F.).}

M a t e ri a l: 1 우 (ad.), Burnside, 21.IV.1932.

Tholymis tillarga wurde in Burnside ein einziges Mal erbeutet. Die Form flog nachts ans Licht. Darin stimmt sie auch in dem Verhalten der Art in Java überein, wo sie ebenfalls verschiedene Male bei Nacht am Lichte gefangen werden konnte.

\section{Pantala flavescens (F.).}

M a t e ri a l: 1 ^ึ, 2 ㅇ, Burnside, 16.-29.IV. und Katherine, 6.V.1931; 1 ㅇ, Burnside, 3.V.1932.

\section{Rhyothemis graphiptera (Ramb.).}

M a t e ri a l : 2 ô, 2 ㅇ, Burnside, 16.IV. und 2.V.1931; 5 ô, Kadarri und Bullrun Swamp, 20.IV.1931; 3 ㅇ, Marrakai, 16.-19. V.1931.

Als besonders auffallend gefärbte Form tritt Rhyothemis graphiptera vor allen übrigen nordaustralischen Libellen stark hervor. Langsamen, schwirrenden und abgzsetzten Fluges bewegt sie sich in den Morgenstunden über den Ufern der Sümpfe und setzt sich oft zu Hunderten an den Uferpflanzen nieder, wo sie leicht erbeutet werden kann. Tagsüber und in der grossen Mittagshitze kann sie auch auf dem Fluge weit im Trockengebiet wahrgenommen werden. Auffallend ist aber ihre besondere Lebhaftigkeit während der kurzen Dämmerung bis in die rasch einbrechende Dunkelheit hinein. So schwärmten grosse Mengen jeweilen in dieser Zeit um einen einzel stehenden, in voller Blüte befindlichen Akazienbaum beim Stationshause von Burnside, kleinere Insekten jagend, welche sich nachts in erhöhtem Masse zum Blütenbesuche einfanden.

32. Hydrobasileus brevistylus (Brauer).

M a t e ri a l: 2 ô, 1 우 (ad.), Marrakai, 17. und 21. IV. 1931.

33. Tramea loewi auct. (? nec. Brauer).

? 1866. Brau er, Verh. zool. bot. Ges. Wien, 16, p. 563-564. -- ô Ceram (T. löwii). 
? 1913. Ris, Cat. Coll. Selys Libell., fasc. 16, p. 975-977. - Kei, Aroe, New Guinea, Australia,? pars (T. löwii).

1926. Tillyard, Ins. Austr. New. Zealand, p. 86 , pl. 4, fig. 8 (q Insekt). - Australia (T. löwii).

M a t e ri a l : 1 ๙ิ, 3 ㅇ, Darwin, 14.IV.1931; $1 \hat{\jmath}, 1$ ㅇ, Shoe-

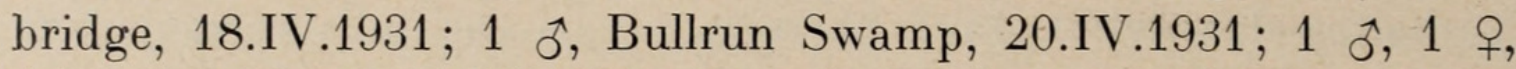
Adelaide River, 21.IV.1931; 1 ô, 1 o, Marrakai, 17.-20.V.1931; 1 ô, Katherine, 5.VI.1931.

Die indo-australischen Arten der Gattung Tramea sind revisionsbedürftig. In meiner Bearbeitung der papuanischen Libellenfauna hoffe ich das Bruchstück einer möglichst genauen Durcharbeitung des umfangreichen Tramea-Materiales dieser und benachbarter Gegenden zu geben, und bei dieser Gelegenheit auch die (ursprünglich von Ceram beschriebene) $T$. loewi des australischen Festlandes mit einzubeziehen.

Ich möchte jetzt schon darauf hinweisen, dass die Brauer'sche loeswi m. E. mit der viel besser bekannten loeswi Australiens in keiner näheren Beziehung steht und sich aller Wahrscheinlichkeit nach dem Formenkreise limbata Desj. enger anschliessen dürfte.

Tramea loewi ist eine in Nordaustralien recht häufige Form, die oft weit abseits vom Wasser angetroffen wird. Sie fliegen oft, wie dies Pantala fiavescens auf Java über den Reisfeldern tut, in Schwärmen über den austrocknenden Grasebenen in 4-5 m Höhe und lassen sich von Zeit zu Zeit ruhend an den Spitzen der gewaltigen Grashalme nieder. In der Ruhe wie auch im Fluge fällt die Form sofort durch das grosse karminrote Analfeld auf. Copulation wurde im Mai am Katherin River beobachtet, wobei die Paare sich wild jagen und nach Abnahme der Spermatophoren in der Luft wiederum trennen. Zur Eiablage schwebt das Weibchen über Tümpeln und stehendem Wasser, wobei beim Schlagen mit dem Abdomen zur Eiablage die Oberfläche leicht berührt wird.

34. Macrodiplax cora Brauer.

M a te ri a l: 2 ๙ึ, 1 q (ad.), Marrakai, 16.-19.V.1931. 


\section{Fam. Corduliidae.}

\section{Subfam. Cordulinate.}

\section{Hemicordulia intermedia Selys.}

(Fig. 8.)

1871. Selys, Synopsis des Cordulines, p. 21-22 sep. - ô Queensland1906. Martin, Cat. Coll. Selys, fasc. 17. Cordul., p. 15. - ô Queensland.

1909. Tillyard, Proc. Linn. Soc. N. S. Wales, V. 33 (1908), p. $740-$ 742, pl. 21, fig. 2 (Insekt), 3-4 (Apps.). - ô Cooktown.

M a t e ri a l: 1 ô, 1 우 (ad.), Burnside, 27.IV. und 3.V.1932.

Das Auffinden dieser ausserordentlich seltenen und merkwürdigen Art ist eines der schönsten Resultate der Ausbeute. Das $q$ war noch unbeschrieben.

ㅇ ( A llot y p e). - Dem ô sehr ähnlich. Färbung und Verteilung der dunklen Zeichnungen an Kopf, Thorax und den Beinen in allen Einzelheiten gleich, aus tief gelb und dunkel schwarzblau- und grün metallisch zusammengesetzt.

Flügel zart, Aderung dünn, ganz rötlichgelb, die Costa wie beim $\widehat{0}$ von der proximalen Seite des Pterostigmas bis zur Spitze schwarz. Vorderflügel an der Basis hyalin, Hinterflügel mit sehr kleinem, diffus gelblichem Basisfleckchen bis zur Cuq und Ende der Membranula; diese ist lichtgrau, weisslich gesäumt. Die distale Hälfte beider Flügel, vom Brückenursprung bis etwa 2 Zellen vor der Spitze und einige Zellen am Flügelrande freilassend, tief safrangelb. Pterostigma dem $\hat{\sigma}$ gleich, trüb ockergelb, das distale Viertel ziemlich scharf begrenzt dunkelbraun. Anq $\frac{7}{5}, P n q \frac{5}{7}$.

Abdomen schlank, anfänglich ziemlich breit (Basis des 3. Segmentes, 2,7 mm), bis Ende 4. deutlich verschmälert, weiter bis

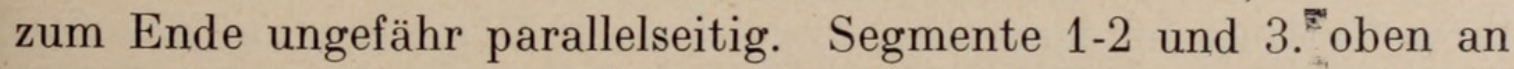
der Basis gewölbt, unten flach. Die seitliche schwarze Binde auf Segment 2 ist in der Mitte schmal durch den queren gelben Mittelfleck unterbrochen; die durchlaufende gelbe Binde jederseits auf dem Rücken des 3. Segments ist etwas breiter und vorn deutlicher 
medianwärts ausladend. 9. Segment oben und seitlich ganz dunkel, 10. hellgelb, mit schwarzer Basislinie (wie beim $\widehat{\jmath}$ ).

Appendices anales schwarz, ziemlich genau um die Hälfte kürzer als beim $\hat{\jmath}$. Valvula vulvae gelblich, dünn und schalenförmig,
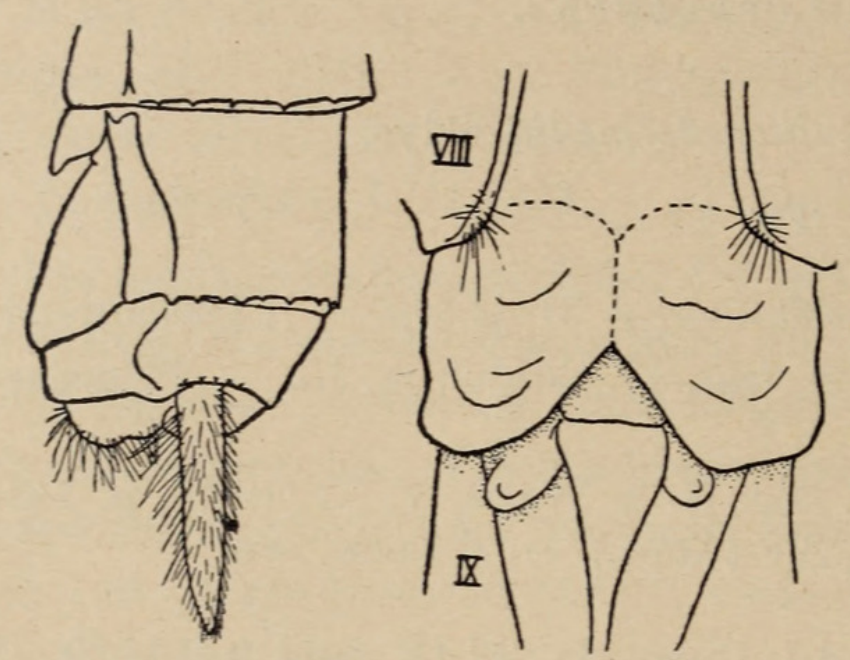

FIG. 8. - Hemicordulia intermedia Selys. ㅇ, Allotype, N. T.

Ende des Abdomens von der linken Seite, und Valvenstruktur von unten, stark vergrössert. unten convex, kaum abstehend. 9. Sternit scharf gekielt, hellgelb (Fig. 8).

Länge: +, Abdomen + Appendices $<29$, Hinterflügel 28,5, Pterostigma, $\frac{1.6}{1.5} \mathrm{~mm}$.

Die Beschreibung des $\hat{0}$ ist bei DE SELYs genügend vollständig, um die Art leicht und rasch zu erkennen. Die eigentümliche Farbenverteilung des Körpers ist aus Tillyards schöner Abbildung ersichtlich. In dieser Zeichnung ist aber die schwarze Medianbinde des Thorax im Vergleich mit dem vorliegenden Pärchen viel zu breit dargestellt. Die Oberseite ist ganz gelb bis auf eine schmale, unten verbreiterte Längsbinde über die Mediannaht. Trotzdem halte ich alle Exemplare für sicher identisch.

Masse des $\sigma^{\hat{*}}$ : Abdomen + Appendices 30, Hinterflügel 28, Pterostigma $\frac{1.8}{1.6} \mathrm{~mm}$.

\section{Fam. Gomphidae.}

36. Ictinus australis Selys.

M a te ri a l: 5 우 (ad.), Marrakai, 16.-21.V.1931.

Diese grosse und leicht kenntliche Art ist der einzige Vertreter ihrer Gattung in Australien und ist nach Tylliard auf das tropische und subtropische Gebiet beschränkt. I. australis kommt in veränderter Form auch in Neu-Guinea und in den nördlichen Molukken (Halmahera) vor, geht aber weiter westlich nicht über Celebes hinaus. 


\section{Fam. Aeschnidae.}

\section{Austrogynacantha heterogena Tillyard.}

1908. Tillyard, Proc. Linn. Soc. N. S. Wales, V. 33, p. 423-431.

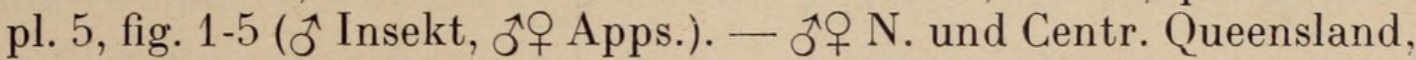

M a t e r i a l : 1 ô (semiad.), Kadarri, 20.IV.1931; 2 오 (juv. und ad.), Burnside, 2. und 28.V.1931.

Die endemische Gattung Austrogynacantha wurde von Tillyard, zweifellos mit Recht, für die einzige aberrante Art heterogena aufgestellt. Beide Geschlechter wurden von diesem Autor sehr ausführlich beschrieben und kenntlich abgebildet. Die Differenzen gegen Gynacantha und Platycantha sind erheblich, und die Verwandtschaftsbeziehungen wurden von TILLYARD anschaulich und im Detail dargelegt.

Unsere Exemplare unterscheiden sich in nichts wesentlichem von den bereits bekannten. Offenbar eine periodisch auftretende und daher seltene Art.

\section{Anax papuensis (Burm.).}

M a t e r i a l: 2 ô, 1 ㅇ (ad.), Katherine, 6.V.1931 und 1 ㅇ, id., 5.VI.1931; 1 우 (ad.), Burnside, 4.V.1932.

In ganz Australien gemein.

Von Anax papuensis wurde am Katherine River die Eiablage beobachtet. Dabei fliegen die Tiere nahe der Wasseroberfläche bis sie ihnen zusagende Wasserpflanzen gefunden haben. Schwirrend tauchen sie dann den fast senkrecht stehenden Hinterleib ins Wasser ein, so dass die Flügel mit der Wasseroberfläche gerade bündig sind, worauf die Eier in das Substrat eingestochen werden. Das $\hat{o}$ hält dabei das , senkrecht stehend, fest.

\section{Anax gibbosulus Ramb.}

1832. Rambur, Hist. Nat. Ins. Névropt., p. 187. - Australia.

1867. Hagen, Zool. bot. Ges. Wien, Bd. 17, p. 41-43. - Celebes

(A. gibbosulus + panybeus).

1898. Krüger, Stett. ent. Zeitg., Bd. 59, p. 271-274. — Sumatra (?), Java (?), Soemba. 
1900. Ris, Arch. f. Naturg., 1, p. 191-192 (Tabelle), Taf. 9, fig. 20 (Apps.). - Kei.

1908. Martin, Cat. Coll. Selys, fasc. 18. Aeschines, p. 24-25, fig. 1 (Flügel), 18 (Apps.). - Kei, Nouvelle Guinée.

1913. Ris, Abh. Senckenb. Naturf. Ges., Bd. 34, p. 526-527. -- ํㅜㅇ Aroe, Ceram.

M a te ri a l: 1 ô (semiad.), Burnside, IV.1931.

Das Zentrum des Verbreitungsgebietes dieser Art scheint im Osten des indo-australischen Archipels zu liegen, wo sie zusammen mit $A$. fumosus Hagen viel häufiger vorkommt als guttatus Burm. Obwohl die Areale dieser drei Arten sich in weitem Umfang decken (das ernorme Verbreitungsgebiet der guttatus reicht von den Seychellen bis nach Fiji mit zahlreichen Stationen im HimalayaGebiet, Südchina, u.s.w.), dominiert guttatus in den Molukken und dem papuanischen Gebiet jedenfalls nicht mehr derart wie z. B. auf den grossen Sunda-Inseln. Ueber Celebes hinaus wird sie tatsächlich auf verschiedenen Molukken-Inseln, wie Halmahera, Buru und Ceram durch $A$. fumosus und $A$. gibbosulus vertreten, während sie von der Insel Bali ostwärts entlang der südlichen Kette der kleinen Sunda-Inseln nur noch sporadisch vorzukommen scheint.

Die Grenzen des Wohngebietes der A. gibbosulus sind nicht bekannt, und einige frühere Angaben bedürfen noch immer der Bestätitgung. Ich möchte einstweilen KR ̈̈GER's Angaben über ihr Vorkommen in Sumatra und Java als un si c he r betrachten, vor alledem weil er von den ihm vorliegenden Stücken sagt: "Grosse Bedenken habe ich auch gegen die gelben Flecke der übrigen Segmente [vom 3. Segment ab gezählt], von denen bei guttatus deutlich 3 vorhanden sind, bei gibbosulus nur 2 vorhanden sein sollen. Ich sehe bei den hiesigen Stücken von gibbosulus bei sehr scharfem Zusehen eine Andeutung von 3 Flecken ». Zweitens aber auch weil eben in Sumatra und Java neben guttatus noch eine zweite, mit fumosus Hag. nahe übereinstimmende und sehr seltene Art vorkommt, die noch nicht genau beschrieben und abgebildet wurde, jedoch gewiss als eine selbständige Spezies zu betrachten ist. Siehe auch Ris (1927). ${ }^{1}$

${ }^{1}$ Ich fing diese sehr ausgezeichnete Art in Mehrzahl an der Wijnkoops Baai, im Süden Javas, wo sie zusammen mit guttatus, aber hauptsächlich in der Abenddämmerung herumfliegt. 
Sehr schöne und recht typische Exemplare der gibbosulus liegen mir heute von den kleinen Sunda-Inseln Sœmba und Flores vor.

Es ergeben sich für gibbosulus also eigentlich nur folgende sichergestellte Stationen: Sœmba, Flores, Celebes (die panybeus Hagen's), Ceram, Kei-Inseln, Aroe-Inseln, südliches Neu-Guinea (teste Risi, andere Ortschaften sind fraglich und beziehen sich wahrscheinlich auf maclachlani Förster), und Nord-Australien (terra typica).

Neben guttatus, gibbosulus, selysi Förster und einer noch unbeschriebenen Art, kommt in Neu-Guinea noch eine fünfte Art, maclachlani Förster, vor, die auf die grosse Insel beschränkt zu sein scheint und von der ich eine sehr grosse Serie aus der Umgebung der Humboldt Bai zu untersuchen imstande war. Sie steht der gibbosulus zweifellos am nächsten, mit der sie folgende Merkmale gemeinsam besitzt:

1. Grosse Arten, Abdomen + Appendices mindestens $70 \mathrm{~mm}$ lang.

2. Oberer Teil der Stirn mit deutlicher schwarzer T-Zeichnung.

3. Hinterflügel an der Membranula hyalin oder kaum gelblich; Flügel mit starker Neigung zum Gelbwerden bei voller Ausfärbung.

4. Abdomen sehr lang und schlank, im 2. Segment sehr stark verengt, von da allmählich verbreitert, die grösste Breite erst im sehr breiten 8. Segment wieder erreichend.

5. Abdomen dunkel; Segmente 3-7 jederseits mit einem Seitenfleck nahe dem Hinterrand und höchstens noch ein kleines Fleckchen auch am Vorderrand von Segment 4-7 (also 3-7, niemals mit mehr als zwei hellen Flecken). Segment 8 jederseits mit nur einem Fleck.

Die folgende Tabelle gibt die wesentlichsten Unterschiede, die zum Teil Ris (l. c., 1900) entnommen sind.

A. gibbosulus. - Appendices superiores äusserst kurz gestielt; der Innenrand distal vom Basalzahn nicht concav und hinter der Mitte fast gerade; Spitze zu einem scharfen, fast geraden Zähnchen der Aussenecke vorgezogen. Mittelkante hoch, vom Innenrand gleichmässig entfernt bleibend.

Pterostigma durchschnittlich bedeutend länger, mindestens 5,2 (bis 6,5) $\mathrm{mm}$. Membranula an der Basis fast immer scharf abgesetzt weiss.

Abdomen nach dem Ende sehr stark erweitert. Segmente 3-7 
jederseits mit ziemlich grossem, rundlichem, hellem Seitenfleck nahe dem Hinterrand, dieser auf 7-9 besonders deutlich (auf 8-9 etwa die Hälfte der Länge einnehmend). Von Segment 4 an auch am Vorderrand noch ein deutliches, aber kleines Fleckchen bis zu Segment 7.

A. maclachlani. - Appendices superiores an der Basis deutlich schmäler und etwas gestielt, der Innenrand distal vom Basalzahn etwas concav und hinter der Mitte ausgebuchtet, der Endteil bedeutend schmäler; Spitze entweder vollständig abgerundet, oder nur mit einer leisesten Andeutung eines stumpfen Zähnchens an der Aussenecke. Mittelkante etwas niedriger, geschwungen, der Innenseite anfänglich näher gerückt.

Pterostigma kurz, meistens ca. $4 \mathrm{~mm}$ und maximal $5 \mathrm{~mm}$ lang. Membranula nahezu vollständig schwarz, an der Basis kaum lichter.

Abdomen noch ein wenig schlanker, terminale Segmente weniger stark erweitert. Segmente 3-7 jederseits nur mit einem ganz kleinen hellen Seitenfleck nahe dem Hinterrand, 8-9 mit etwas grösserem (aber doch noch sehr kleinen!) Fleck.

Das einzige Männchen von Burnside stimmt vorzüglich mit einer kleinen Serie gleichen Geschlechts von der Insel Sœmba überein, ist aber weniger ausgefärbt und daher im allgemeinen viel lichter, mit deutlicher ausgeprägter Fleckenzeichnung. Die Masse sind folgende: Abdomen + Appendices 72, Hinterflügel 55,5 , Pterostigma $\frac{5.2}{5.5} \mathrm{~mm}^{1}$.

Neben A. papuensis und gibbosulus kommt in Nord-Australien als dritte Art auch noch $A$. guttatus vor.

Anax gibbosulus ist wohl eine der grössten und am schwierigsten $\mathrm{zu}$ fangenden nordaustralischen Libellen. Sie fliegt namentlich über grösseren stehenden Gewässern und zeigt in den Abendstunden recht erhöhte Tätigkeit. Das einzige Tier, welches gefangen werden konnte, wurde in den frühen, kühlen Morgenstunden erbeutet, nachdem es aus seiner Ruhe aufgestört worden war. Im Fluge oder über den Sumpfflächen wird es kaum möglich sein, der sehr wachsamen Tiere habhaft zu werden.

1 Ganz neuerdings erhielt ich von Miss Cynthia Longfield (London) noch eine briefliche Mitteilung über ihre Reise durch den malayischen Archipel (1929-1930), worin sie mir u. a. freundlichst mitteilt, während ihres kurzen Besuches auf dem kleinen Koralleninselchen Kisar (Kisser, nordöstlich von Timor) im Dezember 1929 einige Männchen der Anax gibbosulus erbeutet zu haben. Für die Erlaubnis, die Beobachtung an dieser Stelle erwähnen zu dürfen, spreche ich Miss LANGField meinen besten Dank aus. 


\section{ZITIERTE LITERATUR.}

1932. Lieftinck, M. A. The Dragonflies (Odonata) of New Guinea and neighbouring Islands. Part I. Descriptions of new genera and species of the families Lestidae and Agrionidae. Nova Guinea15, Zool. 5 (1932); p. 485-602 (1-118 sep.), Figs.

1911. Ris, F. Cat. Coll. Selys Longchamps, Fasc. 13. Libellulinen, 5 (1911), p. 529-700, Fig.

1913. Ris, F. Odonaten von den Aru- und Kei-Inseln gesammelt durch Dr. H. Merton, 1908, nebst Uebersicht über die von den AruInseln bekannten Odonaten. Abh. Senckenb. Naturf. Ges. 34 (1913), p. 503-536, T. 23.

1916. Ris, F. H. Sauter's Formosa Ausbeute. Odonata. Supplem. Entom. 5 (1916), p. 81, Fig.

1927. Ris, F. Odonaten von Sumatra, gesammelt von Edward Jacobson. Zool. Mededeel. Leiden 10, 1 (1927): 1-49, Fig.

1917. Sıöstedt, Y. Results of Dr. E. Mjöberg's Swedish Scientific Expeditions to Australia, 1910-1913. 16. Odonaten. Arkiv för Zool., V. 11, 11 (1917), p. 1-44, Taf. 1-4.

1906. Tillyard, R. J. New Australian species of the family Agrionidæ, (Neuroptera: Odonata). Proc. Linn. Soc. New South Wales 1906, T. 31, 2 (1906), p. 177-194, Pl. 17.

1913. Tillyard, R. J. On some new and rare Australien Agrionidae (Odonata). Ibid., 1912, V. 37, 3 (1913), p. 404-479, Pls. 44-49.

1913. Tillyard, R. J. Description and Life-History of a new species of Nannophlebia. Ibid., 1912, 37, 4 (1913), p. 712-726, Pl. 74.

1926. Tillyard, R. J. On a collection of Papuan Dragonflies (Odonata) made by the late Mr. Allan McCulloch in 1922-3, with descriptions of new species. Rec. Austral. Mus., 15, 2 (1926), p. 157-166, Figs. 


\section{$2 \mathrm{BHL}$ Biodiversity Heritage Library}

Lieftinck, A. 1933. "Odonaten aus Nordaustralien." Revue suisse de zoologie 40, 409-439. https://doi.org/10.5962/bhl.part.117662.

View This Item Online: https://www.biodiversitylibrary.org/item/148564

DOI: https://doi.org/10.5962/bhl.part.117662

Permalink: https://www.biodiversitylibrary.org/partpdf/117662

\section{Holding Institution}

American Museum of Natural History Library

\section{Sponsored by}

BHL-SIL-FEDLINK

\section{Copyright \& Reuse}

Copyright Status: In copyright. Digitized with the permission of the rights holder.

Rights Holder: Muséum d'histoire naturelle - Ville de Genève

This document was created from content at the Biodiversity Heritage Library, the world's largest open access digital library for biodiversity literature and archives. Visit BHL at https://www.biodiversitylibrary.org. 